\title{
Aconitine Intoxication Misdiagnosed as Acute Myocardial Infarction
}

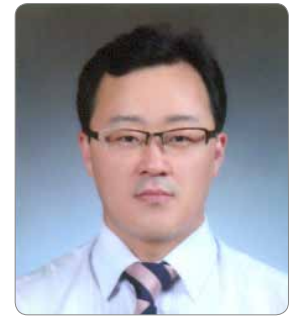

Min-Soo Ahn, MD

Division of Cardiology Yonsei University Wonju College of Medicine, Wonju, Republic of Korea

Received: March 3, 2016

Revision Recieved: April 19, 2016

Accepted: June 29, 2016

Correspondence: Min-Soo Ahn, MD

Division of Cardiology Yonsei University Wonju

College of Medicine 20, Ilsanro, Wonju,

Gangwon 26426, Republic of Korea

Tel: +82-33-741-0917

Fax: +82-33-741-1219

E-mail: ahnn0102@medicus.co.kr

Copyright (C) 2016 The Official Journal of Korean Heart Rhythm Society Editorial Board and MMK

Communications Limited

\begin{abstract}
A 55-year-old woman was admitted because of sudden onset of chest tightness after ingesting a herbal medicine. She experienced refractory ventricular arrhythmia after admission. Electrocardiography showed ST-segment abnormalities that mimicked acute myocardial infarction, but the coronary artery was found to be normal. After conservative management, the cardiac rhythm was stabilized. This was an unusual case of aconitine intoxication that mimicked acute myocardial infarction.
\end{abstract}

Key Words: - Aconitine -Myocardial Infarction -Tachycardia, Ventricular

\section{Introduction}

In Asia, aconitine poisoning is common because of the continued use of aconite roots in traditional medicine as an analgesic, anti-inflammatory, and cardiotonic agent. Aconitine and other Aconitum alkaloids are highly poisonous cardio-toxins and neurotoxins present in all parts of the plants of the Aconitum genus (aconite). The major toxin in these plants is aconitine, which interacts with the voltage-gated sodium channels.

Here, I report an unusual case of acute aconitine intoxication manifesting as chest tightness and ST-segment elevation that mimicked acute myocardial infarction (AMI).

\section{Case}

A 55-year-old woman was presented to the emergency department with due to chest tightness during sleep at 01:00. She had no medical history. Initial blood pressure was recorded at
100/33 mmHg. At baseline electrocardiography (ECG), nonsustained narrow QRS tachycardia and premature ventricular contraction (PVC) were observed (Figure 1A). 5 minutes after admission to the emergency room, rhythm was changed into ventricular tachycardia occurred along with altering morphologies (Figure 1B). At 30 minutes, wide QRS tachycardia was observed and blood pressure was recorded at $92 / 49 \mathrm{mmHg}$. 3 times of electrical cardioversion (200 J) performed. but failed to achieve conversion to normal sinus rhythm (Figure 2). Subsequently, blood pressure went further down to $60 / 40 \mathrm{mmHg}$ along with deterioration of mental status. The patient then received mechanical ventilatory support along with intravenous infusion of amiodarone and vasopressors. On follow-up ECG, rhythm changed to narrow $\mathrm{QRS}$ tachycardia, but ST elevation was profound on aVR, and ST depressed further on $\mathrm{V}_{2-6}, \mathrm{I}$, II, and aVL (Figure 3). Initial CK-MB and troponin-I levels were $58 \mathrm{ng} /$ $\mathrm{mL}(<3.7 \mathrm{ng} / \mathrm{mL})$ and $0.025 \mathrm{ng} / \mathrm{mL}(<0.046 \mathrm{ng} / \mathrm{mL})$, respectively. On the initial echocardiography, the regional wall 
A
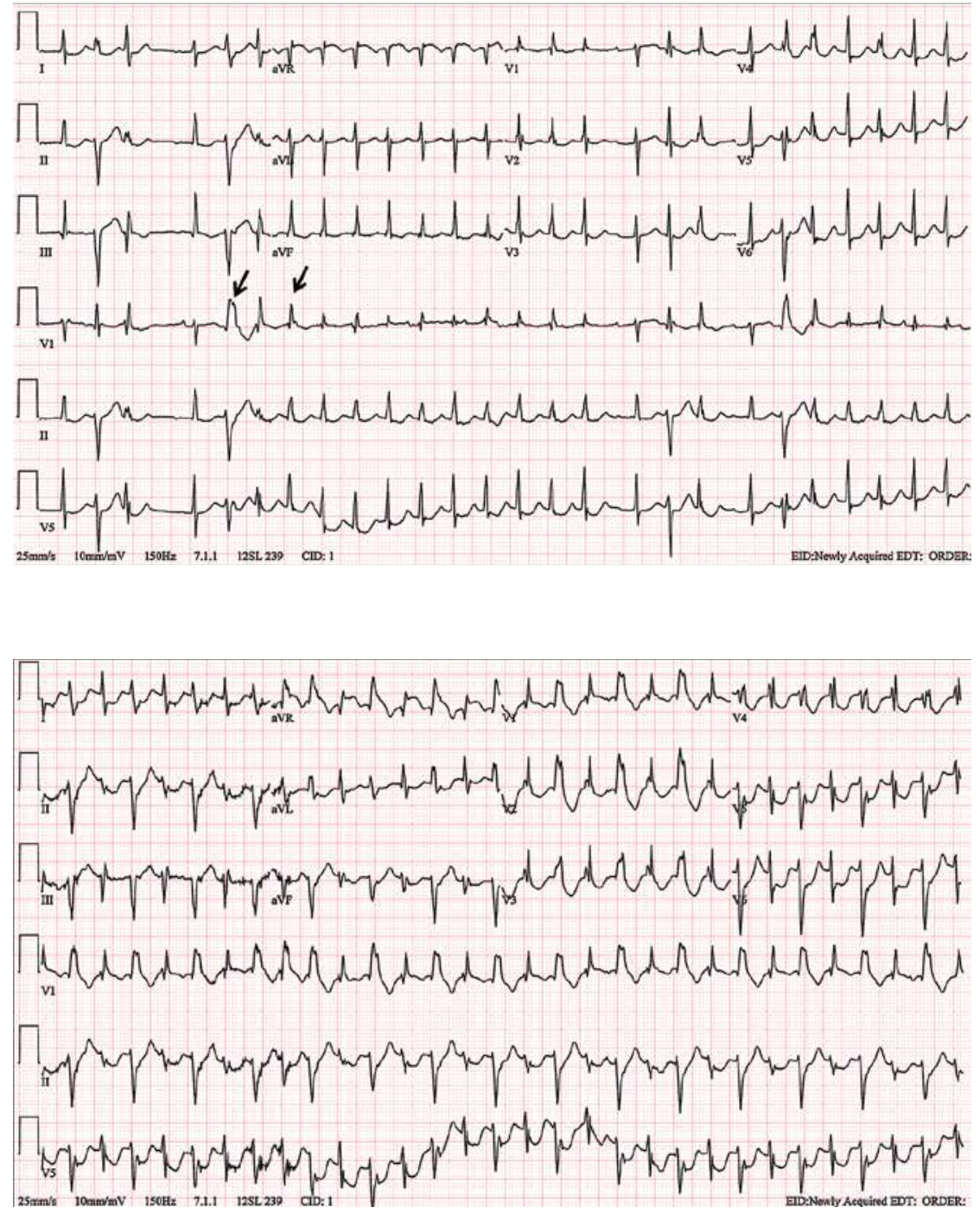

Figure 1. (A) Twelve-lead electrocardiography in the emergency department showing non-sustained narrow QRS tachycardia and premature ventricular contraction. (B) Follow-up electrocardiography showing ventricular tachycardia with alternating morphology.

motion abnormality could not be evaluated precisely because of tachycardia. As previous reports, ST-segment elevation in lead aVR is a predictor of left main disease or 3-vessel disease in patients with non-ST-segment elevation acute coronary syndromes. Generally, ST-segment elevation in lead aVR is reciprocal to and accompanied by ST-segment depression in the precordial leads, which is also a predictor of left main disease or 3-vessel disease. ${ }^{1}$ Therefore, we assumed that the patient might have severe coronary artery disease and ventricular tachycardia probably due to ischemia. However, coronary angiography revealed no stenosis. Thus, after angiography, we obtained the patient's precise medical history from her daughter. The daughter told us that the patient had received a herbal medicine called "Bujasasim-tang" that contains Aconitum carmichaelii Debeaux, which the patient had taken on the previous night. Consequently, her rhythm returned to normal without the need for specific management (Figure 4). Echocardiography revealed no regional wall motion abnormality. The patient was discharged 3 days later without any complications. 


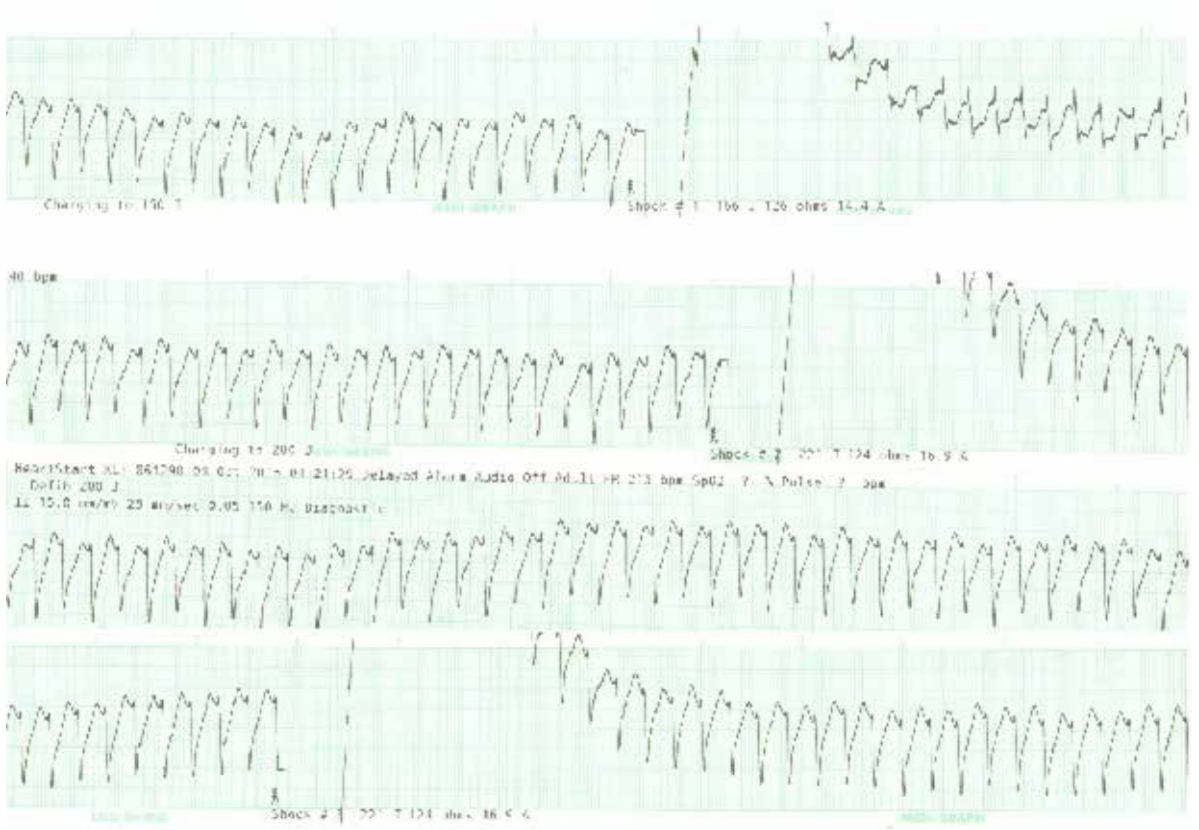

Figure 2. Rhythm strip during the three interventions with direct current cardioversion.

\section{Discussion}

Aconitine and other Aconitum alkaloids fall within the categories of highly poisonous cardiotoxins and neurotoxins. They are present in all parts of the plants within Aconitum the genus (aconite), most abundantly in the roots and root tubers. Aconite poisoning is rather common in Asia because of the widespread use of aconite roots in traditional medicine for treatment of pain, agitation, indigestion, and other conditions.

The cardiotoxicity and neurotoxicity of aconitine and related alkaloids is due to their interactions with voltage-sensitive sodium channels in the cell membranes of excitable tissues, including myocardium, nerves, and muscles. ${ }^{2}$ Aconitine and mesaconitine bind with high affinities to the open state of voltage-sensitive sodium channels, blocking their inactivation, thereby leading to persistent activation of the channels. As a consequence of continuous sodium influx and sustained depolarization, the sodium channels become refractory to excitation. ${ }^{3}$ Aconitine can induce ventricular ectopy, ventricular tachycardia, torsades de pointes, ventricular fibrillation, and mortality in a dose-dependent manner. The electrophysiological mechanism responsible for arrhythmia induction is the triggered activity due to delayed afterdepolarization and early after-depolarization. Aconitine binds to sodium channels and prolongs their open state, favoring $\mathrm{Na}^{+}$ influx into the cytosol. The associated increase in intracellular calcium via $\mathrm{Na}^{+}-\mathrm{Ca}^{2+}$ exchange system further induces triggered activity. Moreover, the arrhythmogenic properties of aconitine are partly due to its cholinolytic (anticholinergic) effects and block the vagus effect ${ }^{4}$

The cardiovascular manifestations include hypotension, palpitations, chest pain, bradycardia, sinus tachycardia, ventricular ectopy, ventricular arrhythmias (tachycardia, torsade de points, and/or fibrillation), and junctional rhythm. Ventricular arrhythmias are most likely to occur within the first 24 hours during severe poisoning. Aconite-induced bidirectional tachycardia, atrioventricular dissociation, and pulmonary edema have also been reported. In some patients, elevated plasma creatine kinase activity without ECG evidence of acute myocardial 


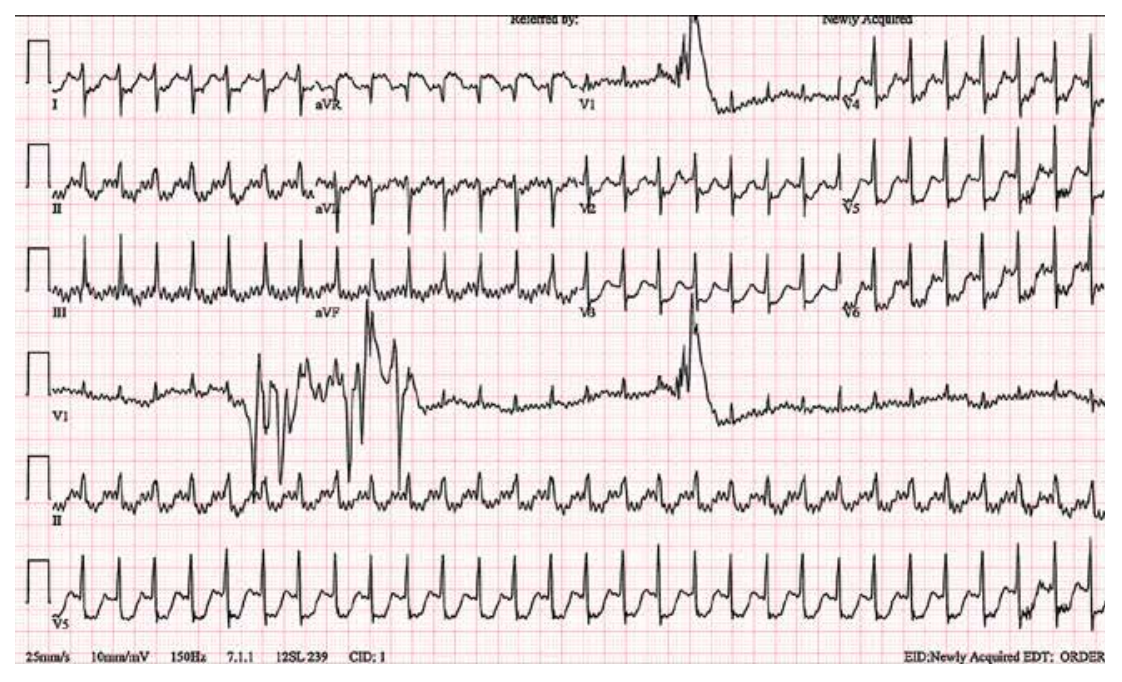

Figure 3. Follow-up electrocardiography showing significant ST-segment deviation.

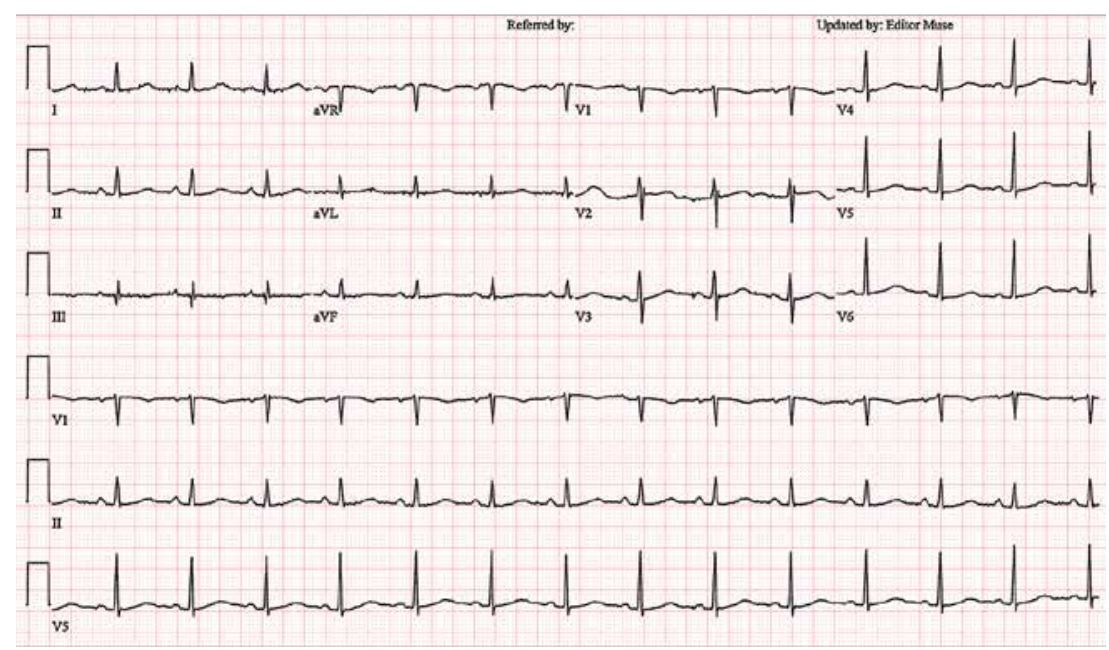

Figure 4. Follow-up electrocardiography showing normal sinus rhythm.

infarction has also been reported. ${ }^{5}$ However, in the current case, ECG changes resembled with acute myocardial infarction. The possible mechanism behind ECG changes mimicking myocardial infarction may be that aconitine causes cardiotoxicity by producing arrhythmias, which leads to demand ischemia of the myocardium. Another possible explanation is persistent activation of sodium channels in the myocardium, which allows excessive influx of sodium and calcium ions into the myocardial cells, leading to ionic imbalance. This can further result in uncoupling of mitochondrial oxidative phosphorylation, disruption of cellular enzymes, and cell death, a process analogous to excitotoxicity in the central nervous system. ${ }^{6}$

Overall, this case demonstrates that aconitine intoxication can cause electrocardiographic changes that resemble acute myocardial infarction. 


\section{References}

1) Misumida N, Kobayashi A, Fox JT, Hanon S, Schweitzer P, Kanei Y. Predictive Value of ST-Segment Elevation in Lead aVR for Left Main and/or Three-Vessel Disease in Non-ST-Segment Elevation Myocardial Infarction. Ann Noninvasive Electrocardiol. 2016;21:91-97.

2) Chan TY. Aconite poisoning. Clin Toxicol (Phila). 2009;47:279285

3) Friese J, Gleitz J, Gutser UT, Heubach JF, Matthiesen T, Wilffert B, Selve N. Aconitum sp. alkaloids: the modulation of voltage- dependent $\mathrm{Na}+$ channels, toxicity and antinociceptive properties. Eur J Pharmacol. 1997;337:165-174.

4) Sheikh-Zade YR, Cherednik IL, Galenko-Yaroshevskii PA. Peculiarities of cardiotropic effect of aconitine. Bull Exp Biol Med. 2000;129:365-366.

5) Tai YT, But PP, Young K, Lau CP. Cardiotoxicity after accidental herb-induced aconite poisoning. Lancet. 1992;340:1254-1256.

6) Lin CC, Phua DH, Deng JF, Yang CC. Aconitine intoxication mimicking acute myocardial infarction. Hum Exp Toxicol. 2011;30:782-785. 